\title{
On the Plastic Zone around a Crack Tip in a Rock Specimen
}

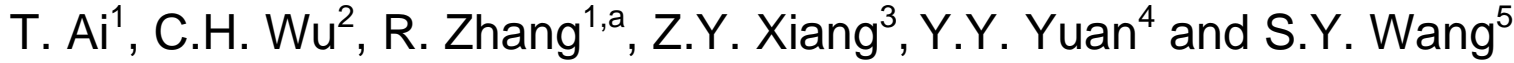 \\ ${ }^{1}$ State Key Laboratory of Hydraulic and Mountain River Engineering, School of Water Resource and \\ Hydropower, Sichuan University, Chengdu 610065, China
}

${ }^{2}$ School of Resource and Environmental Science, Chongqing Univ., Chongqing 400030, China

${ }^{3}$ Changjiang Chongqing waterway engineering Bureau, Chongqing 400000, China

${ }^{4}$ China construction steel Structure corp. LTD., Shenzhen 518000, China

${ }^{5}$ China construction eignth engineering division. corp. LTD., Shanghai 200120, China

azhangru@scu.edu.cn (corresponding author)

Keywords: Mises yield criterion; Stress intensity factor; Plastic zone; Boundary collocation method

\begin{abstract}
The stress intensity factors (SIFs) for a infinite volume were corrected by two dimensionless quantities $Y_{\mathrm{I}}$ and $Y_{\mathrm{II}}$ which were obtained by employing the Boundary Collocation Method (BCM). The expression of the elastic-plastic boundary of a mixed crack for a cracked specimen was formulated based on the Mises yield criterion. Finally, the shape and scale of the plastic zone for tensile-shear cracks and compressive-shear cracks were analyzed and some meaningful conclusions were obtained. The results show that: the shape of a cracked specimen has an significant effect on the plastic zone; the scale of the plastic zone is much smaller than its real scale under the assumption of infinity volume; the orientation angle of a crack affects both the shape and scale of the crack tip plastic zone significantly under tension; the friction between the crack surfaces can reduce the scope of the crack tip plastic zone effectively under compression.
\end{abstract}

\section{Introduction}

Currently, more and more underground engineering projects have been implemented. The investigation on the failure mechanism of deep rock masses has become a hot issue. Under high temperature and pressure condition, the plastic deformation around a crack tip is significant, and this plastic deformation determines crack initiation, propagation and instability [1]. Thus, it is necessary to study the characteristics of the plastic zone under small scale yield condition. Dugdale [2] proposed a D-M model to calculate the size of plastic zone. Jendoubi [3](1991) and Ranganathan [4] (1994) suggested that the shape and size of the plastic zone can be determined by Mises yield criterion. Zhao[5] proposed the effective stress expression and obtained the initial elastic-plastic boundary function around a crack tip. Li [6] introduced the first and second estimations of plastic zone scale in detail. However, the rock specimen used in experimental study was finite, and it is unreasonable to analyze and evaluate the shape and scale of the plastic zone according to the fracture theory based on the assumption of an infinite volume.

\section{Elastic-plastic boundary function}

As is well known, for plain stress and plain strain problems, the stresses can be expressed as

$$
\begin{aligned}
& \sigma_{x x}=\frac{K_{\mathrm{I}}}{\sqrt{2 \pi r}} \cos \frac{\theta}{2}\left(1-\sin \frac{\theta}{2} \sin \frac{3 \theta}{2}\right)-\frac{K_{\mathrm{II}}}{\sqrt{2 \pi r}} \sin \frac{\theta}{2}\left(2+\cos \frac{\theta}{2} \cos \frac{3 \theta}{2}\right), \\
& \sigma_{y y}=\frac{K_{\mathrm{I}}}{\sqrt{2 \pi r}} \cos \frac{\theta}{2}\left(1+\sin \frac{\theta}{2} \sin \frac{3 \theta}{2}\right)+\frac{K_{\mathrm{II}}}{\sqrt{2 \pi r}} \sin \frac{\theta}{2} \cos \frac{\theta}{2} \cos \frac{3 \theta}{2}, \\
& \sigma_{x y}=\frac{K_{\mathrm{I}}}{\sqrt{2 \pi r}} \cos \frac{\theta}{2} \sin \frac{\theta}{2} \sin \frac{3 \theta}{2}+\frac{K_{\mathrm{II}}}{\sqrt{2 \pi r}} \cos \frac{\theta}{2}\left(1-\sin \frac{\theta}{2} \sin \frac{3 \theta}{2}\right),
\end{aligned}
$$




$$
\sigma_{z z}=\left\{\begin{array}{ll}
0 & , \text { for plane stress } \\
v\left(\sigma_{x x}+\sigma_{y y}\right) & \text { for plane strain }
\end{array} .\right.
$$

where $v$ is the Poisson's ratio. By employing the well-known Mises yield function, the elastic-plastic boundary function can be obtained as

$$
r=1 /(8 \pi C) \cdot\left(b_{11} K_{\mathrm{I}}^{2}+2 b_{12} K_{\mathrm{I}} K_{\mathrm{II}}+b_{22} K_{\mathrm{II}}^{2}\right) .
$$

where $C$ was obtained by yield stress $\sigma^{\mathrm{YS}}$ which determined through a directional tensile test, $b_{11}=\xi(1+\cos \theta)+\sin ^{2} \theta, \quad b_{12}=\sin 2 \theta-\xi \sin \theta, \quad b_{22}=1+\xi(1-\cos \theta)+3 \cos ^{2} \theta, \quad \xi=2\left(1-2 v^{*}\right)^{2} / 3$, and $v^{*}=0$ for plane stress problems, $v^{*}=v$ for plane strain problems.

For a finite scale specimen, Eq.2 can be expressed as

$$
r=1 /(8 \pi C) \cdot\left(c_{11} K_{\mathrm{I}}^{2}+2 c_{12} K_{\mathrm{I}} K_{\mathrm{II}}+c_{22} K_{\mathrm{II}}^{2}\right) \text {. }
$$

where $c_{11}=Y_{\mathrm{I}}^{2} b_{11}, c_{12}=Y_{\mathrm{I}} Y_{\mathrm{II}} b_{12}, c_{22}=Y_{\mathrm{I}}^{2} b_{22}, Y_{\mathrm{I}}$ and $Y_{\mathrm{II}}$ are dimensionless stress intensity factors.

Under compression, the crack is a mode II crack. The effective shear stress along the crack is

$$
\tau_{\mathrm{e}}=\sigma_{\mathrm{S}}-f \sigma_{\mathrm{N}} .
$$

where $\sigma_{\mathrm{S}}=\sigma_{1} \cos ^{2} \beta+\sigma_{3} \sin ^{2} \beta$ is the shear stress component along the crack and $\sigma_{\mathrm{N}}=\left(\sigma_{1}-\sigma_{3}\right) \sin \beta \cos \beta$ is the normal stress component, $\sigma_{1}$ is the axial compression load, $\sigma_{3}$ is the confining pressure, $a$ is the half-length of the crack, $\beta$ is the angle of the crack and the axis of $\sigma_{3}$, and $f$ is the friction coefficient. If the condition $f<\tan \beta$ is satisfied, then the elastic-plastic boundary function of finite size specimen under pressure load can be taken as

$$
r=1 /(8 \pi C) \cdot\left[1+\xi(1-\cos \theta)+3 \cos ^{2} \theta\right] Y_{\mathrm{II}}^{2} K_{\mathrm{II}}^{2} .
$$

\section{Solution of the dimensionless stress intensity factors}

Boundary collocation method (BCM) has been widely used in fracture mechanics [7-8]. For linear fracture mechanics problems, the stresses are related to these functions by following relations [9].

$$
\begin{aligned}
& \sigma_{x x}+\sigma_{y y}=4 \operatorname{Re} \Phi(z), \\
& \sigma_{y y}-i \tau_{x y}=\Phi(z)+\Omega(\bar{z})+(z-\bar{z}) \overline{\Phi^{\prime}(z)}, \\
& X+i Y=-i[\varphi(z)+\omega(\bar{z})+(z-\bar{z}) \overline{\Phi(z)}]_{A}^{B}, \\
& \Phi(z)=\varphi^{\prime}(z), \Omega(z)=\omega^{\prime}(z), \\
& \omega(z)=z \varphi^{\prime}(z)+\psi(z) .
\end{aligned}
$$

The stress boundary conditions of the crack surfaces can be transformed to a Hilbert problem [8], therefore, $\Phi(z)$ and $\Omega(z)$ can be calculated by the following expressions

$$
\begin{aligned}
& \Phi(z)+\Omega(z)=\left(2 \sum_{k=1}^{M} A_{k} z^{k-1}\right) / \sqrt{z^{2}-a^{2}} . \\
& \Phi(z)-\Omega(z)=2 \sum_{k=1}^{M} B_{k} z^{k-1},
\end{aligned}
$$

where $M$ is the number of summation terms, $A_{k}$ and $B_{k}$ are complex constants. From Eqs.9 and 10, $\varphi(z), \omega(z), \varphi^{\prime}(z)$ and $\omega^{\prime}(z)$ can be expressed as

$$
\begin{aligned}
& \varphi(z)=\sqrt{z^{2}-a^{2}} \sum_{k=1}^{M} E_{k} z^{k-1}+\sum_{k=1}^{M} F_{k} z^{k}, \\
& \omega(z)=\sqrt{z^{2}-a^{2}} \sum_{k=1}^{M} E_{k} z^{k-1}-\sum_{k=1}^{M} F_{k} z^{k},
\end{aligned}
$$




$$
\varphi^{\prime}(z)=\sum_{k=1}^{M} E_{k}\left[z^{k} / \sqrt{z^{2}-a^{2}}\right] \cdot\left[k-(k-1)(a / z)^{2}\right]+\sum_{k=1}^{M} k F_{k} z^{k-1},
$$

Inserting Eqs.13, 14 and 15 into Eq.8, the resultant force boundary condition can be written as

$$
-Y+i X=\sum_{k=1}^{M} E_{k}\left[\sqrt{z^{2}-a^{2}} z^{k-1}+\sqrt{z^{2}-a^{2}} \bar{z}^{k-1}\right]+\sum_{k=1}^{M} F_{k}\left(z^{k}-\bar{z}^{k}\right)+\overline{\left\{\frac{1}{\sqrt{z^{2}-a^{2}}} \sum_{k=1}^{M} E_{k}\left[k-(k-1)\left(\frac{a}{z}\right)^{2}\right] z^{k}+\sum_{k=1}^{M} k F_{k} z^{k-1}\right\}}(z-\bar{z}) \text {. }
$$

The crack tip stress intensity factors are

$$
K_{\mathrm{I} 0}-i K_{\mathrm{II} 0}=2 \sqrt{\pi} \sum_{k=1}^{M} E_{k} a^{k-3 / 2},
$$

and the dimensionless stress intensity factors are

$$
Y_{\mathrm{I}}-i Y_{\mathrm{II}}=\sum_{k=1}^{M} \operatorname{Re}\left(E_{k}\right) a^{k-2} / K_{\mathrm{I}}-i \cdot 2 \sum_{k=1}^{M} \operatorname{Imag}\left(E_{k}\right) a^{k-2} / K_{\mathrm{II}} \text {. }
$$

In order to obtain the coefficients $E_{k}$ and $F_{k}$, a certain number of points along the specimen boundary were used [7-8]. By employing Eq.16, the coefficients can be computed.

\section{Shape of the plastic zone in a specimen}

For the uniaxial tension/compression problems, the elastic-plastic dimensionless boundary function can be written as

$$
R=\left(\sigma_{y} / \sigma_{1}\right)^{2} r / W=(3 / 8) \cdot(a / W) \cos ^{2} \beta\left(b_{11} \cos ^{2} \beta+2 b_{12} \sin \beta \cos \beta+b_{22} \sin ^{2} \beta\right) .
$$

where $\sigma_{1}$ is uniaxial tensile/compression load, $\sigma_{y}$ is the yield strength. According to Eq.3 the modified expression of the elastic-plastic boundary function is

$$
R=\left(\sigma_{y} / \sigma_{1}\right)^{2} r / W=(3 / 8) \cdot(a / W) \cos ^{2} \beta\left(c_{11} \cos ^{2} \beta+2 c_{12} \sin \beta \cos \beta+c_{22} \sin ^{2} \beta\right) .
$$

The elastic-plastic boundary function under compression is given by

$$
R=\left(\sigma_{y} / \sigma_{1}\right)^{2} r / W=(3 / 8) \cdot(a / W) \cos ^{2} \beta\left[c_{22}\left(\sin \beta \cos \beta-f \cos ^{2} \beta\right)^{2}\right] .
$$

In the following analysis, the Poison's ratio $v$ is set to 0.25 , the width of the specimen is $2 \mathrm{~W}$, and the length ratio, $a / W$, is arranging in 0.1 and 0.35 . For the uniaxial tension problems, $\beta$ varies from $0^{\circ}$ to $66^{\circ}$. For the uniaxial compression problems, $\beta$ is $45^{\circ}$ and $f=\{0,0.25,0.5$ and 0.75$\} . R_{x}$ and $R_{y}$ is the parallel and perpendicular components of the plastic zone boundary respectively.

For uniaxial tension problems, the results, as shown in Fig.1, indicate that the shape and the scale of the plastic zone around the crack tip calculated by using Eq.19 and 20 are almost the same where ratio $=0.1$. However, the scale calculated by using Eq.20 is much larger than the scale obtained according to Eq.19 where ratio $=0.35$. From Fig.1, it can be seen that the orientation angle of the crack affects both the shape and the scale of the plastic zone significantly, and the scale of the plastic zone decreases with the orientation angle.

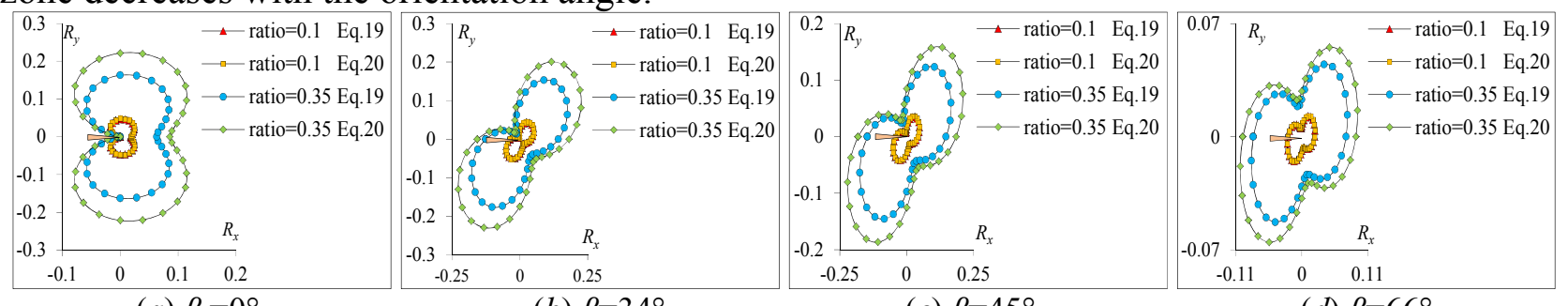
(a) $\beta=0^{\circ}$
(b) $\beta=34^{\circ}$
(c) $\beta=45^{\circ}$
(d) $\beta=66^{\circ}$

Fig.1 Plastic zone for crack angle is $0^{\circ}, 34^{\circ}, 45^{\circ}, 66^{\circ}$ respectively. 
The results, calculated by applying Eq.19 and Eq.21, are shown in Fig.2. It can be seen that the result calculated by using Eq.19 is close to the result calculated by using Eq. 21 where the length of the crack is much smaller than that of the specimen, and the plastic zone scale calculated by employing Eq. 21 is much larger than the scale calculated by Eq. 19 where the ratio is 0.35 . It also can be seen that the shape of the crack tip plastic zone doesn't change although different friction coefficients were used. However, the scale decreases with the friction coefficients.

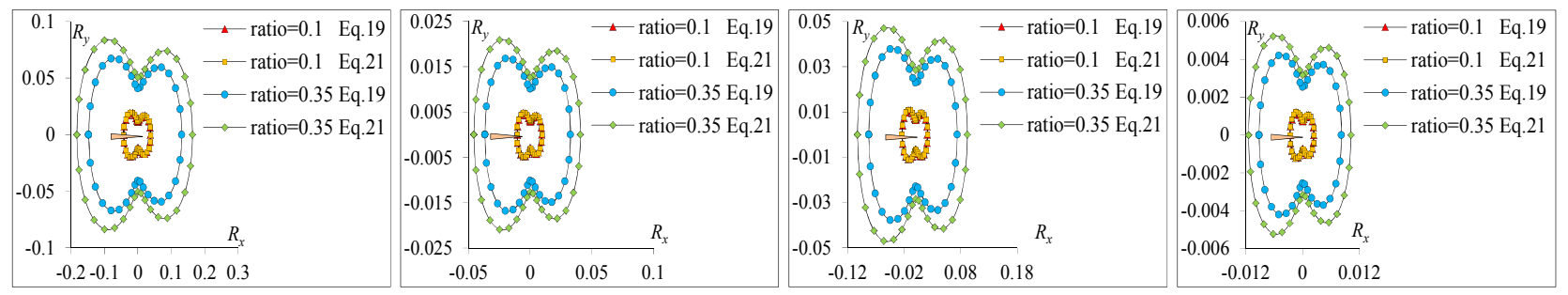

(a) $f=0$ (b) $f=0.25$ (c) $f=0.5$ (d) $f=0.75$

Fig.2 Plastic zone for friction coefficient is $0,0.25,0.5,0.75$ respectively.

\section{Summary}

In this paper, the analytic expression of the elastic-plastic boundary around a crack tip was derived by employing BCM and Mises yield criterion, and the shape and scale of the plastic zone under tensile/compressive loading conditions are analyzed respectively. Based on the above analysis, the following conclusions can be obtained:

1) The size of a specimen has a significant effect on both shape and scope of the plastic zone.

2) Under tension, the crack angle affects the crack tip plastic zone significantly.

3) Under compression, the shapes of the crack tip plastic zone with different friction coefficients are the same, and the friction can reduce the plastic deformation effectively.

\section{Acknowledgements}

This work was financially supported by the Sichuan Youth Science \& Technology foundation (2012JQ0031).

\section{References}

[1] Wang W.J., Zhao N.L.: China Petroleum Machinery, Vol.18(1990) , p.16 20.(In Chinese)

[2] Dugdale D.S.: J.Mech.Phys.Solids, Vol.8(1960), p.100-115.

[3] Jendoubi K., Ranganathan N., Merah, N.: J. Testing Evaluation, Vol.19(1991), p.201-209.

[4] Ranganathan N., Jendoubi K., Merah N.: J. Eng. Mater. Technol,Vol.116(1994), p.187-192.

[5] Zhao Y.S.: Journal of Mechanical Strength, Vol.11(1992), p.51-53. (In Chinese).

[6] Li S.Y., He T.M., et al.: Introduction of Rock Fracture, Chinese Science and Technology University Press, Hefei(2010). (In Chinese).

[7] Zhu Z, Ji S, Xie H: Engng Fract Mech, Vol.54(1996), p.731-741.

[8] Zhu Z, Xie H, Ji S: Engng Fract Mech, Vol.56(1997), p.647-655.

[9] N.I Muskelishvih: Some basic problems of mathematical theory of elasticity, Noordhoff International Publishing, Leyden (1975). 